\title{
Retrospective analysis of diffuse interstitial lung dis- ease in 10 hospitals in Tianjin
}

\author{
SHOUCHUN PENG ${ }^{1}$, LUQING WEI ${ }^{1}$, JIE CAO ${ }^{2}$, GUIYING LIU ${ }^{3}$, YANPING LAI ${ }^{4}$,

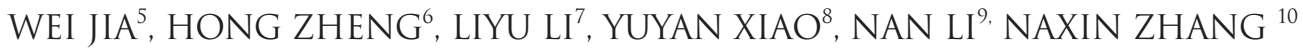

\section{Abstract}

Objective: To investigate the constituent ratio and clinical features of diffuse interstitial lung disease (DILD) in Tianjin, and to identify the existing problems of its diagnosis and treatment.

Methods: Data of all patients diagnosed as DILD in ten comprehensive hospitals in Tianjin from 2003 to 2009 was collected. The disease constituent ratio of DILD to all kinds of diseases in the respiration department or in the whole hospital, the clinical manifestation, lung function, the result of blood gas analysis, and biopsy were analyzed.

Results: A total of 804 DILD patients were included in the study aged (69.28 12.09$)$ years with a male/female ratio of 1.32 . The disease constituent ratios of DILD were $4.52 \%$ in respiratory department and $0.31 \%$ in the whole hospital in 2003, which increased to $35.9 \%$ ond $1.51 \%$ respectively in 2009. Reticular abnormality (43.1\%), ground glass (25.6\%) and honeycombing (11.4\%) were the predominant imaging manifestations. 26.7\% patients' lung function were tested, and $10.3 \%$ had diffusion function tests. Most patients presented with hypoxemia, and the average oxygenation index was (281 \pm 98.3$) \mathrm{mmHg}$. Only $4.0 \%$ of patients underwent bronchoalveolar lavage, and 3.5\% had pathologic data.

Conclusion: The disease constituent ratio of DILD increased progressively from 2003 to 2009 . A definite diagnosis of DILD demands cooperation of physicians, radiologists and pathologists. Doctors should attach more importance on lung function test, bronchoalveolar lavage and biopsy.

Key words: Lung disease, interstitial, Epidemiology, Constituent ratio

\section{Introduction}

Diffuse interstitial lung disease (DILD) refers to a group of lung diseases characterized by fibrosis and inflammation in the interstitium and parenchyma of the lung. Prolonged DILD may result in pulmonary fibrosis, which can result in death from respiratory failure. There are more than 150 diseases in the spectrum of DILD, and the incidence rate of DILD has been increasing in recent years. However, the study on the incidence of DILD is rare in China. So this study is aimed to analyze the incidence and clinical manifestation of DILD retrospectively, which may help to improve the clinical diagnosis and treatment of DILD.

\section{Methods \\ Participants}

A total of 804 patients diagnosed as DILD in ten comprehensive hospitals in Tianjin from 2003 to 2009 were included, ages between 19 97 years, among which 457 patients were
1. Department of Respiration Medicine, Affiliated Hospital Medical College of CAPF, Tianjin 300162, China

2. Department of Respiration Medicine, ,Tianjin Medical University General Hospital, Tianjin 300052, China

3. Department of Respiration Medicine, First Teaching Hospital of Tianjin University of Traditional Chinese Medicine , Tianjin 300193, China

4. Department of Respiration Medicine, the Second Hospital of Tianjin Medical University, Tianjin 300211, China

5. Tianjin Chest Hospital, Tianjin, 300051, China

6. Department of Respiration Medicine, Tianjin First Center Hospital, Tianjin 300192, China

7. Department of Respiration Medicine, Tianjin Fourth Center Hospital, Tianjin 300140, China 8.Department of Respiration Medicine, Tianjin Gongan Hospital, Tianjin 300042, China

9.Department of Respiration Medicine, Tianjin Third Hospital, Tianjin 300250, China

10.Department of Respiration Medicine, Tianjin Third Central Hospital, Tianjin 300170, China

CORRESPONDING AUTHOR: Luqing Wei

Department of Respiration Medicine, Affiliated Hospital Medical College of CAPF, Tianjin 300162,China luqing-wei@163.com

Funding: This research received no specific grant from any funding agency in the public, commercial, or not-for-profit sectors. 
male. The chest X-ray and/or CT tests of all patients showed the changes of DILD and parenchymal infiltration. The diseases included seven diseases of idiopathic interstitial pneumonia (IIP), lymphangioleiomyomatosis (LAM), Langerhan-cell granulomatosis (LCG), pulmonary fibrosis due to connective tissue disease, sarcoidosis, extrinsic allergic alveolitis (EEA), pulmonary eosinophilia, diffuse panbronchiolitis (DPB), and other unclassified interstitial lung diseases and occupational diseases. The cases were excluded if pulmonary fibrosis was caused by tumor, infectious disease, or cardiac disease.

\section{Diagnostic criteria}

The diagnosis criteria included: International Multidisciplinary Consensus Classification of the Idiopathic Interstitial Pneumonias (IIP) published by American Thoracic Society (ATS) and European Respiratory Society (ERS) in 2002 [1]; Guideline on Diagnosis and Management of Idiopathic Pulmonary Fibrosis (IPF) issued in China in 2002 [2]; the guidelines on diagnosis and management of diffuse parenchymal lung disease publish by British Thoracic Society (BTS) [3] and those published on the Chinese textbooks of internal medicine; and the evidence-based guidelines for diagnosis and management of IPF issued by ATS/ERS/JRS/ALAT in 2011[4].

\section{Data collection}

The following information was collected from study participants: demographic information such as gender, ethnicity, and age; the course of the disease; length of hospital stay, symptoms and signs when admitted; results of chest X-ray, CT, pulmonary function test and blood gas analysis; the methods for definite diagnosis; and the relative treatment.

\section{Statistical analysis}

All data were analyzed with SPSS 13.0.

\section{Results}

General information and the disease spectrum

A total of 804 patients were included in this study, among which 6 were Hui ethnic, 3 were Man ethnic, and the rest were all Han ethnic. The average age was $69.28 \pm 12.09$ years, with 14 patients aged under 40 years old, 34 aged between 41 and 50 years, 125 aged between 51-60, 177 aged between 6170, 322 aged between 71- 80, and 132 aged above 80 years. There were 457 male patients, with the average age of $69.89 \pm 12.02$ years and 347 female, with age of $68.49 \pm 12.15$ years. 444 patients were smokers. The mean course of the disease was 3.24 years, and the average length of hospital stay was 15.5 days. And 82 patients died in hospital, which accounted for $10.2 \%$ of all patients.

The disease distribution was as follows: 466 unclassified cases (55.5\%); 197 cases of IPF (24.5\%) aged $(70.17 \pm 11.17)$ years with male/ female ratio of $1.75 ; 24$ cases of other IIPs (3.0\%) including 6 cases of cryptogenic organizing pneumonia (COP), 5 cases of acute interstitial pneumonia (AIP) and 13 cases of nonspecific interstitial pneumonia (NSIP); 78 cases due to connective tissue disease, including 58 cases of rheumatoid arthritis, 2 cases of derma- tomyositis, 1 case of ankylosing spondylitis, 8 cases of Sjogren's syndrome, 3 cases of systemic lupus erythematosus (SLE), and 6 cases of mixed connected tissue disease; 47 cases due to other disease, including 25 with silicosis, 3 with asbestosis , 5 with pneumoconiosis, 11 with drug induced pneumonitis, 2 with radiation pneumonitis, 1 with neurofibroma; and 12 cases of rare ILD, including 4 cases of sarcoidosis, 1 case of EEA, 1 case of eosinophilic pneumonia, 1 case of pulmonary alveolar proteinosis (PAP), 2 cases of LCG, 2 cases of DPB, and 1 case of alveolar hemorrhage.

\section{The changes of hospitalization rate over the decade}

In general, the constituent ratio of DILD increased year by year. The patients with DILD accounted for $4.52 \%$ of the inpatients in the respiratory department and $0.31 \%$ in the entire hospital in 2003, which increased to $39.5 \%$ and $1.51 \%$ in 2009, respectively. There were 29 DILD inpatients in 2003 (4.52\% in respiration department, $0.31 \%$ in the whole hospital), 55 in 2004 (7.38\%o, 0.46\%o), 57 in 2005 (7.27\%o, 0.47\%), 92 in 2006 (11.2\%o, 0.72\%o), 125 in 2007 (14\%o, $0.87 \%$ ), 209 in 2008 (22.4\%o, 1.34\%o), and 237 in 2009 (39.5\%o, 1.51\%o).

\section{Clinical features}

All patients experienced cough and breathlessness in varying degrees. Velero rales were heard in 457 (56.8\%) patients, which were especially common in IPF patients. Clubbing fingers or toes were also manifested in $127(15.8 \%)$ patients. 


\section{OF ORIGINAL RESEARCH}

\section{Imaging examinations}

Chest x-rays were taken of only 192 patients, while all 804 patients undertook CT scan, of which 33 took Highresolution CT scan (HRCT). The imaging examinations showed that the main lung features of the cases in our study were reticular abnormality (347 cases, $43.2 \%$ ), ground glass change (206 cases, $25.6 \%$ ), nodular change (79 cases, $9.8 \%$ ) and honeycomb change (92 cases, $11.4 \%)$.

\section{Pulmonary function}

Pulmonary function tests were used in 215 patients $(26.7 \%)$, among which 83 patients' lung diffusion capacities were tested. Among the 215 patients, the total lung capacity (TLC\%pred) was $(70.01 \pm 20.55) \%$; the forced expiratory volume in $1 \mathrm{~s}$ (FEV1\%pred) was $(61.12 \pm 25.87) \%$; the forced vital capacity (FVC\%pred) was $(60.16 \pm 20.74) \%$; the diffusing capacity of the lung for carbon monoxide (DLCO\%pred) was $(47.51 \pm 23.42) \%$; and the residual volume (RV\% pred) was $(89.93 \pm 96.23) \%$.

\section{Arterial blood gas analysis}

There were 429 patients (53.4\%) who had arterial blood gas tests. The mean oxygenation index $(\mathrm{PaO} 2 / \mathrm{FiO} 2)$ was $281.0 \pm 98.3 \mathrm{~mm} \mathrm{Hg}(1 \mathrm{~mm} \mathrm{Hg}=0.133$ $\mathrm{kPa}$ ), and the mean arterial carbon dioxide tension $(\mathrm{PaCO} 2)$ was $39.6 \pm 10.4 \mathrm{~mm}$ $\mathrm{Hg}$. Two hundred and thirty six patients (29.4\%) suffered Type I respiratory failure, and 40 (5.0\%) suffered Type II respiratory failure.

\section{Pathological tests}

Thirty two cases $(4.0 \%)$ underwent bronchoalveolar lavage, among which one case happened in 2004, 5 in 2006, 9 in 2007, and 17 in 2008. Lung biopsy was done in 28 patients (3.5\%), among which 1 case happened in 2003, 2 in 2004, 2 in 2005, 4 in 2006, 5 in 2007 , and 14 in 2008. Nineteen patients were examined by bronchoscope, of which 1 case was diagnosed as EEA, 1 as PAP, and the rest not classified. Two patients were examined by video-assisted thoracoscopic surgery (VATS), of which one case was diagnosed as sarcoidosis, and the other one not classified. Four patients were examined with thoracotomy, of which one case was diagnosed with NSIP, one as COP, one as UIP, and one as alveolar hemorrhage. Three patients undertook skin biopsy, of which one was diagnosed as sarcoidosis, one as Sjogren's syndrome, and one as ankylosing spondylitis.

\section{Treatment}

Among the 804 patients, 222 patients $(27.6 \%)$ were treated with glucocorticoids, $5(0.6 \%)$ were treated with glucocorticoids and cytotoxic drugs, 48 (6.0\%) were treated with glucocorticoids and $\mathrm{N}$-acetylcysteine (NAC), 3 were treated with glucocorticoids combining with cytotoxic drugs and NAC, and 15 (1.9\%) were treated only with NAC.

\section{Discussion}

\section{General information}

This study found that DILD patients were mainly adults. The mean age at diagnosis was $69.28 \pm 12.09$ years, while the male to female ratio was 1.32. All patients suffered dyspnea in varying degrees, $56.8 \%$ patients showed the signs of velcro rales, and $15.8 \%$ had clubbing of fingers or toes, which is consistent with the study in Chongqing [5] and inconsistent with the studies in other countries.

The incidence rate of IPF in our study was higher in senior males, with the male/female ratio of 1.75 , which is consistent with Coultas's finding[6]. In our study, the average age of onset of IPF was 70 years, while the other studies showed it was between 40-60 years old [7]. Rudd et al. [7] found that the onset age of IPF was 54 years old ranging between 20 to 73 years with the male/ female ratio of 1.70. Thomeer et al. [8] found that $49 \%$ patients were first diagnosed as IPF at the age of 60 to 79 years, and $39 \%$ were diagnosed at the age of 40-59 years. The course of disease before definite diagnosis was 18 months in our study, while it was 6 months in other countries. And 80\% IPF patients showed velcro rales and $63 \%$ patients had clubbing of fingers and toes.

Among all the cases of pneumoconiosis, silicosis accounted for $76 \%$. Most of these patients were male, most likely because those jobs with high silicate exposure in China are performed by males. For $80 \%$ of patients, the age of onset was 50 years old, which might because it usually takes 20-30 years to develop into silicosis after silicate exposure.

The onset age of pulmonary fibrosis due to rheumatoid arthritis usually ranges between $50-70$ years old. The main body sign is dyspnea, while clubbing fingers or toes and velcro rales are also the common symptoms. In our study, the average age of patients with pulmonary fibrosis due to rheumatoid arthritis was 
68 years, among which $51 \%$ patients had clubbing of fingers and toes, and $81 \%$ showed velcro rales.

\section{The distribution of diseases}

The studies on epidemiology of DILD in America, Germany, Italy, and Mexico [6,9-11] showed that DILD occurred mainly in adults; and IPF, sarcoidosis, and EEA were the most common in the spectrum of DILD. The incidence rate of EEA was relatively low in Italy compared to those in the other three countries; the incidence rate of IPF was relatively low in America; and the incidence rate of sarcoidosis and EEA was relatively low in Mexico, where the unclassified pulmonary fibrosis were more common.

In our study, in the majority of disease entities of DILD, a male predominance was found. Similar to the situation in Mexico, unclassified pulmonary fibrosis took the highest proportion among all diseases, followed by IPF and pneumoconiosis. The percentage of unclassified pulmonary fibrosis was $55.5 \%$ in our study, while the incidence rate and prevalence were $30.9 \%$ and $29.1 \%$, respectively in the other countries [8]. This could be related to the doctors' understanding of DILD and the rare use of pathological technique in diagnosis in China. In our study, the diagnosis was proven by pathology in only $3.5 \%$ of the cases.

Among all cases in our study, the percentage of IPF was $24.5 \%$, which approximated to $20 \%$ as reported in Thomeer et al.'s study [8]. Since there is no effective treatment, it's very important to increase the patients' cognition on IPF.

Among all cases in our study, the percentage of pulmonary fibrosis due to connective tissue disease was $9.7 \%$, and that of interstitial lung disease due to rheumatoid arthritis was $7.2 \%$, while the percentages in other countries were $15 \%$ [12] and 7\% [8] respectively. The rates in China are lower than other countries, which might be related to the patients' characteristics, the diagnostic method of rheumatism, and the doctors' understanding of rheumatism, as we collected the data in departments of respiration medicine instead of rheumatic departments. The initial symptom of most connective tissue disease is pulmonary fibrosis, and then other symptoms will show up gradually, so the patients with connective tissue disease will be first treated in department of respiration medicine, which calls the doctor's attention. To avoid erroneous diagnosis, immunological tests should be regular procedure for the patients in the respiration department, and chest X-ray and pulmonary function testing should be regular tests for patients in the Rheumatology Department, while chest CT scans, bronchoalveolar lavage and biopsies should be done when necessary. The proportion of ILD caused by inhalation of dust has decreased in China, which shows that the sense of occupational health protection is increasing in China. The proportion of EEA and sarcoidosis were high in other studies in China and other countries, however, the proportions of these two diseases were relatively low in our study, which may indicate their low incidence rates in Tianjin.

\section{The increasing incidence rate of DILD}

The research showed that the incidence rate of DILD increased 8 times from 3.62 per 100,000 person-years in south of Spain [13] to 31.5 per 100,000 personyears in males and 26.1 per 100,000 person-years in females in the state of New Mexico in the United States of America [6]. Also Kornum et al. [14] found that the incidence rate of DILD increased from 27.14/ 100,000 in 1995 to $34.34 / 100,000$ in 2002. What's more, these studies were all done by physicians in respiratory departments, which may underestimate the incidence rate. In our study, the disease constituent ratios of DILD in respiratory departments and the entire hospital all increased, which may suggest that the incidence of DILD is really increasing, or that the highsensitive or new techniques, especially HRCT, lead to improved diagnoses. The research in Demark [15] showed also that the increased application of CT scan increased the possibility of the diagnosis of mild DILD and influenced the classification of DILD.

\section{Laboratory examination}

In our study, the main lung features of DILD patients were reticular change, ground glass change, nodular change and honey comb change in the lung, and the former two changes were most common, which is consistent with other studies. The lung function of DILD patients is also impaired, usually shown as restrictive ventilation impairment with diffusion function impairment. Some patients might have mixed ventilation function disturbance; for example, the patients with pulmonary fibrosis caused by pneumoconiosis and heavy smoke may suffer chronic obstructive pulmo- 
nary disease (COPD). Generally, the blood gas analysis of DILD patients shows Type I respiratory failure, and Type II respiratory failure may appear in the late course of DILD or in patient with DILD with airway obstruction. In this study, we had similar findings that Type I respiratory failure took up $29.4 \%$, while Type II respiratory failure took up $5.0 \%$.

\section{Problems in diagnosis and manage- ment of DILD}

Regular examinations should be increased. The pulmonary function, HRCT, bronchoalveolar lavage, and bronchoscope are important examinations in the diagnosis and identification of DILD; however, the percentage of the patients undergoing each examination accounted for only $1 / 4,4.1 \%, 4.0 \%$ and $2.4 \%$ respectively among all cases, indicating that more importance should be attached on these examinations.

The biopsy rate should be improved. In our data, no more than $10 \%$ of the cases did biopsy, among which $81.82 \%$ were still not clearly diagnosed. The VATS and thoracotomy were only applied in less than $5 \%$ cases. All of these illustrate that more biopsy should be encouraged, and there is still much to learn about the pathological changes of DILD.

The understanding of DILD should be enhanced. In our study, the duration between symptom presentation and definite diagnosis ranged between 1 week and 3.9 years, with an average of 3.24 years, which suggests that it is still difficult for doctors in China to diagnose DILD.

The treatment should be standard- ized. As the guideline released in 2009 [16] has recommended, IPF patients should be treated with glucocorticoids combined with low dose of cytotoxic drugs and NAC instead of glucocorticoids combined only with low dose of cytotoxic drugs. However, only 3 cases followed the guideline.

\section{Acknowledgements}

The study was done with the joint efforts of ILD team in Tianjin of Chinese society of Respiratory disease of Chinese Medicine Association.

\section{Competing interests}

The authors declared no competing interests.

\section{References}

1. American Thoracic Society, European Respiratory Society. American Thoracic Society/European Respiratory Society International Multidisciplinary Consensus Classification of the Idiopathic Interstitial Pneumonias. Am J Respir Crit Care Med 2002; 165: 277-304.

2. Chinese society of Respiratory disease of Chinese Medicine Association. Guideline on Diagnosis and Management of Idiopathic Pulmonary Fibrosis (IPF) (Trail version). Chin J Tuberc Respir Dis 2002; 25(7): 387-389.

3. Raghu G, Collard HR, Egan JJ, Martinez FJ, Behr J, Brown KK, et al. An Official ATS/ERS/JRS/ALAT Statement: Idiopathic Pulmonary Fibrosis: Evidence-based Guidelines for Diagnosis and Management. Am J Respir Crit Care Med 2011; 183(6):788-824.

4. The Diffuse Parenchymal Lung Disease Group of British Thoracic Society. The diagnosis, assessment and treatment of diffuse parenchymal lung disease in adults.Thorax 1999; 54(S1):S1-S28.

5. Yang XM, Wang CZ, Cui SH, Liu Z, Li
XJ, Du XZ. Retrospective analysis of diffuse interstitial lung disease in five teaching hospitals in Chongqing, Chinese Journal of Respiratory and Critical Care Medicine 2008; 7(7):120-123.

6. Coultas DB, Zumwalt R, Black W. The epidemiology of interstitial lung diseases. Am Respir Crit Care Med 1994; 150(4): 967-972.

7. Rudd RM, Haslam PL, Turner-Warwick M. Cryptogenic fibrosing alveolitis: Relationships of pulmonary physiology and bronchoalveolar lavage to response to treatment and prognosis.Am Rev Respir Dis 1981; 124(1):1-8.

8. Thomeer M, Demedts M, Vandeurzen K, VRGT Working Group on Interstitial Lung Diseases. Registration of interstitial lung diseases by 20 centres of respiratory medicine in Flanders. Acta Clin Belg 2001; 56(3):163-172.

9. Roelandt M, Demedts M, Callebaut W, Coolen D, Slabbynck H, Bockaert J, Kips J, Brie J, Ulburghs M, De Boeck K. Epidemiology of interstitial lung diseases in Flanders: registration by pneumologists in 1992-1994. Acta Clin Belg 1995; 50 (5):260-268.

10. Schweisfurth H, Costabel U, Kropp R, et al.Mitteilung der wissenschaftliches Arbeitsgemeinschaft furdie Therapie von Lungenkrankheiten (WATL): Deutsches Fibroseregister miternsten Ergebnissen.Pneumologie 1996; 50:899-901.

11. Rizzato G, Bariffi F. Inchiesta epidemiologica sulle interstiziopatic polmonari in Italia: Datierisultati a macchia dileopardo. Internista 1999; 7:20-24.

12. Strange C, Highland KB. Interstitial lung disease in the patient who has connective tissue disease.Clin Chest Med 2004; 25 (3):549-559.

13. Lopez-Campos JL, Rodriguez-Becerra E, Neumosur Task Group, Registry of Interstitial Lung Diseases. Incidence of interstitial lung diseases in the south of Spain 1998-2000: the RENIA study. Eur J Epidemiol 2004; 19(2):155-161. 
14. Kornum JB, Christensen S, Grijota M, Pedersen L, Wogelius P, Beiderbeck A, Sørensen, HT. The incidence of interstitial lung disease 1995-2005: a Danish nationwide population-based study. BMC Pulm Med 2008; 4:8-24.
15. Raghu G, Nyberg F, Morgan G. The epidemiology of interstitial lung disease and its association with lung cancer. $\mathrm{Br}$ J Cancer 2004; 91(S2): S3-10.

16. Bradley B, Branley HM, Egan JJ, Greaves MS, Hansell DM, Harrison
NK, et al. Interstitial lung disease guideline: the British Thoracic Society in collaboration with the Thoracic Society of Australia and New Zealand and the Irish Thoracic Society.Thorax 2008; 63(S5):v1-58.

\section{Idiopathic pulmonary fibrosis: the diagnosis and management of suspected idiopathic pulmonary fibrosis}

Idiopathic pulmonary fibrosis is a chronic, progressive fibrotic interstitial lung disease of unknown origin. It is a difficult disease to diagnose and often requires the collaborative expertise of a consultant respiratory physician, radiologist and histopathologist to reach a consensus diagnosis. Most people with idiopathic pulmonary fibrosis experience symptoms of breathlessness, which may initially be only on exertion. Cough, with or without sputum, is a common symptom. Over time, these symptoms are associated with a decline in lung function, reduced quality of life and ultimately death.

The median survival for people with idiopathic pulmonary fibrosis in the UK is approximately 3 years from the time of diagnosis. However, about $20 \%$ of people with the disease survive for more than 5 years. The rate of disease progression can vary greatly. A person's prognosis is difficult to estimate at the time of diagnosis and may only become apparent after a period of careful follow-up.

This guideline contains recommendations on the diagnosis of idiopathic pulmonary fibrosis and delivery of care to people with idiopathic pulmonary fibrosis, from initial suspicion of the disease and referral to a consultant respiratory physician, to best supportive care and disease-modifying treatments.

The guideline will assume that prescribers will use a drug's summary of product characteristics to inform decisions made with individual patients.

This guideline recommends some drugs for indications for which they do not have a UK marketing authorisation at the date of publication, if there is good evidence to support that use. The prescriber should follow relevant professional guidance, taking full responsibility for the decision. The patient (or those with authority to give consent on their behalf) should provide informed consent, which should be documented. See the General Medical Council's Good practice in prescribing and managing medicines and devices for further information. Where recommendations have been made for the use of drugs outside their licensed indications ('off-label use'), these drugs are marked with a footnote in the recommendations.

This guidance has been incorporated into the following NICE Pathways, available at http://pathways.nice.org.uk/pathways/ idiopathic-pulmonary-fibrosis, along with other related guidance and products. 